\title{
Impact of the Eulerian chaos of magnetic field lines in magnetic reconnection
}

M.-C. Firpo, W. Ettoumi, A. F. Lifschitz, A. Retinò, R. Farengo, H. E. Ferrari, and P. L. García-Martínez

Citation: Physics of Plasmas 23, 122905 (2016); doi: 10.1063/1.4972544

View online: http://dx.doi.org/10.1063/1.4972544

View Table of Contents: http://aip.scitation.org/toc/php/23/12

Published by the American Institute of Physics

\section{Articles you may be interested in}

Parallel electric fields are inefficient drivers of energetic electrons in magnetic reconnection

Physics of Plasmas 23, 120704120704 (2016); 10.1063/1.4972082

Dynamo-driven plasmoid formation from a current-sheet instability

Physics of Plasmas 23, 120705120705 (2016); 10.1063/1.4972218

Effect of collisions on the two-stream instability in a finite length plasma

Physics of Plasmas 23, 122119122119 (2016); 10.1063/1.4972543

Secondary fast reconnecting instability in the sawtooth crash

Physics of Plasmas 24, 012102012102 (2017); 10.1063/1.4973328

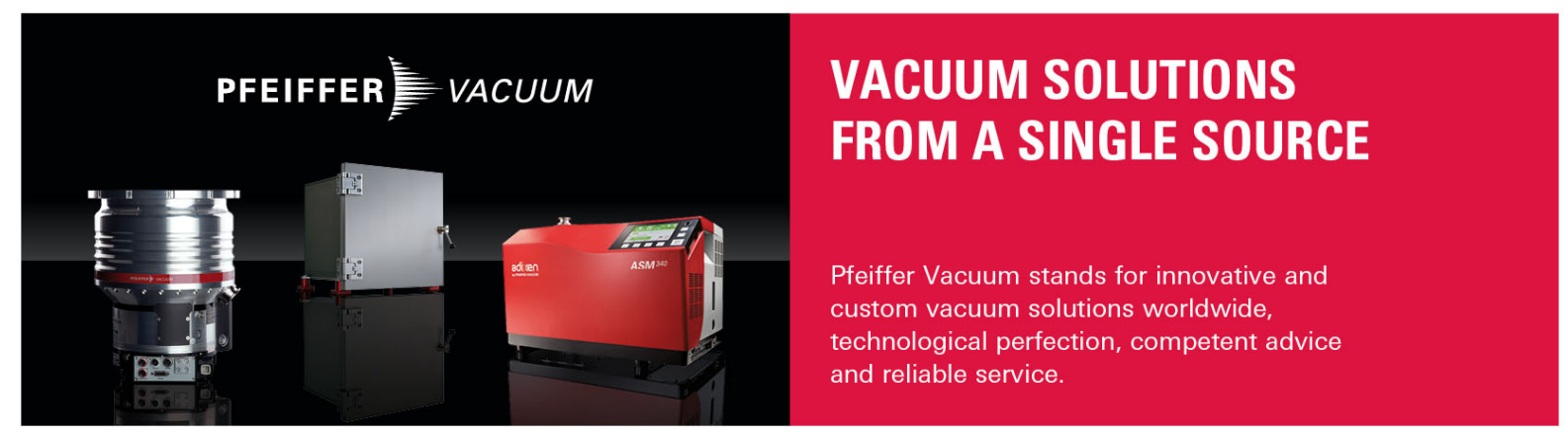




\title{
Impact of the Eulerian chaos of magnetic field lines in magnetic reconnection
}

\author{
M.-C. Firpo, ${ }^{1}$ W. Ettoumi, ${ }^{1,2}$ A. F. Lifschitz, ${ }^{3}$ A. Retinò, ${ }^{1}$ R. Farengo, ${ }^{4}$ H. E. Ferrari, ${ }^{4,5}$ \\ and P. L. García-Martínez $z^{5}$ \\ ${ }^{1}$ Laboratoire de Physique des Plasmas, CNRS - Ecole Polytechnique, 91128 Palaiseau cedex, France \\ ${ }^{2}$ Université de Genève, GAP-Biophotonics, Chemin de Pinchat 22, 1211 Geneva 4, Switzerland \\ ${ }^{3}$ Laboratoire d'Optique Appliquée, ENSTA, CNRS, Ecole Polytechnique, 91761 Palaiseau, France \\ ${ }^{4}$ Centro Atómico Bariloche (CNEA) and Instituto Balseiro (UNC-CNEA), San Carlos de Bariloche, RN 8400, \\ Argentina \\ ${ }^{5}$ Consejo Nacional de Investigaciones Científicas y Técnicas (CONICET), Bariloche, Argentina
}

(Received 5 October 2016; accepted 5 December 2016; published online 23 December 2016)

\begin{abstract}
Stochasticity is an ingredient that may allow the breaking of the frozen-in law in the reconnection process. It will first be argued that the non-ideal effects may be considered as an implicit way to introduce stochasticity. Yet there also exists an explicit stochasticity that does not require the invocation of non-ideal effects. This comes from the spatial (or Eulerian) chaos of magnetic field lines that can show up only in a truly three-dimensional description of magnetic reconnection since the two-dimensional models impose the integrability of the magnetic field lines. Some implications of this magnetic braiding, such as the increased particle finite-time Lyapunov exponents and increased acceleration of charged particles, are discussed in the frame of tokamak sawteeth that forms a laboratory prototype of spontaneous magnetic reconnection. A justification for an increased reconnection rate with chaotic vs. the integrable magnetic field lines is proposed. Moreover, in 3D, the Eulerian chaos of the magnetic field lines may coexist with the Eulerian chaos of velocity field lines, that is more commonly named the turbulence. Published by AIP Publishing.

[http://dx.doi.org/10.1063/1.4972544]
\end{abstract}

\section{INTRODUCTION}

Seventy years ago, the concept of reconnection was suggested by Giovanelli ${ }^{1}$ to explain the particle acceleration in solar flares. Since then, the mechanism of magnetic reconnection has been a remarkably active and puzzling subject of research.

Magnetic reconnection can be defined, in a minimal way, as a sudden rearrangement of the magnetic field that converts the magnetic energy into plasma energy. It is indeed now thought to be at the core of many space physics phenomena as the trigger that releases the energy from the magnetic field. ${ }^{2,3}$ For instance, the magnetic reconnection has been identified to be the underlying mechanism behind the complex astrophysical phenomena such as the solar flares, magnetospheric substorms, ${ }^{4}$ or gamma-ray bursts. Apart from those space manifestations, ${ }^{7}$ magnetic reconnection has also become a major concern in magnetic confinement fusion devices. It is at play in a generic phenomenon of tokamak plasmas known as sawteeth, diagnosed for the first time in the early 1970s. ${ }^{5}$ In this regime, quantities like the core plasma density or temperature undergo a slow rise before suddenly crashing on a periodic basis, so that the resulting time trace of these quantities resembles the edge of a saw. The crash phases coincide with a rearrangement of the magnetic field lines and the expulsion of heat and particles from the plasma core. The basic picture of the sawtooth magnetic reconnection frame is represented in Figure 1. This sawtooth regime, in which the central tokamak plasma suffers almost a periodic abrupt heat and particle rearrangements along with impulsive magnetic field reorganizations, can be considered as a prototype of finite- $B$ (or general) magnetic reconnection, since the toroidal magnetic field may be viewed as a guide field.

Some fundamental issues remain to be clarified and understood to fully unveil the mechanisms behind the magnetic reconnection. ${ }^{6}$ In particular, for a magnetic reconnection to be possible, the electrons have at some point to get free from magnetic slavery, according to the von Steiger's formulation ${ }^{6}$ so that the frozen-in law may be broken. Yet the reason why and how this may happen is unclear. In this article, it will be argued that stochasticity may be considered as one possible ingredient through which this may be realized in the magnetic reconnection process.

Two ways of having stochasticity in the magnetic field will be discussed. First, it will be argued that non-ideal effects may be considered as a "hidden" way to introduce stochasticity. However, there is some accumulating experimental and numerical evidence that this effect may not by itself prove sufficient to account for the fastness of magnetic reconnection. Then, the remaining part of this study will be devoted to the role and importance played by another source of stochasticity, namely, by the spatial chaos of magnetic field lines. This will be shown to necessitate a three-dimensional magnetic description. The farreaching impact of magnetic field lines being chaotic will be illustrated by numerical simulations of test bed models of tokamak sawteeth. It will be shown that the particle acceleration about the crash (reconnection) phase is more efficient and realistic in the case with spatially stochastic magnetic field lines. 


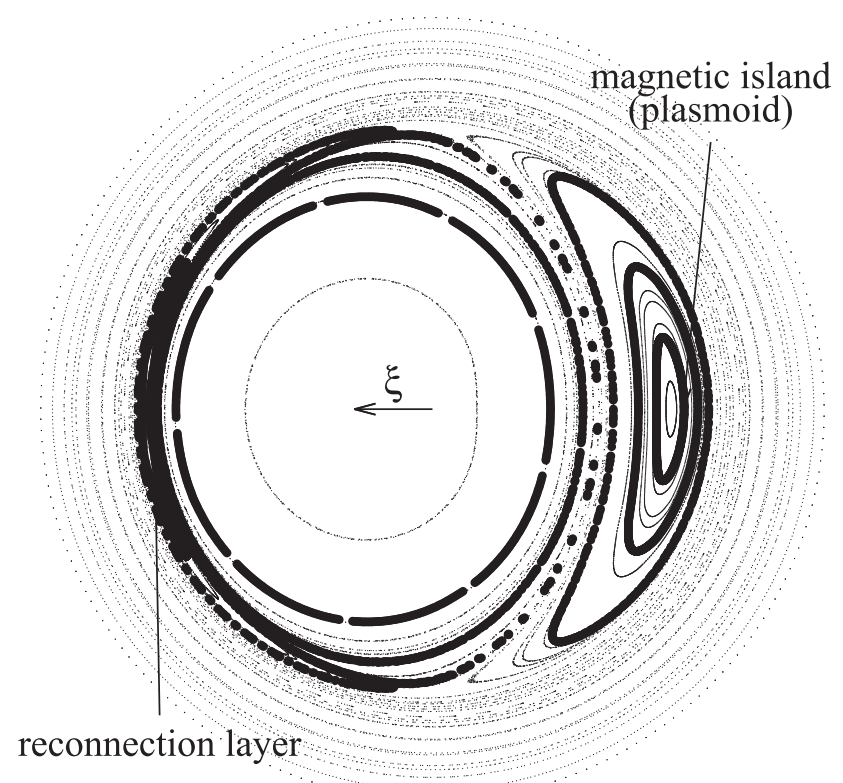

FIG. 1. In certain tokamak operational conditions, axisymmetry breaks and a macroscopic magnetic island is linearly destabilized resulting in a change of the magnetic field topology. This is a well-known laboratory occurrence of spontaneous magnetic reconnection. The early (linear) stage is represented in the figure showing some snapshot of the intersection of magnetic field lines with some tokamak poloidal cross-section. Some points are highlighted to emphasize the region of separatrices.

\section{NON-IDEAL EFFECTS AND STOCHASTICITY}

Neglecting the displacement current in the MaxwellAmpère equation compared to the electric current due to moving charges and using Ohm's law, which is recalled to be a phenomenological relationship stating the proportionality of the electric current and electric field in the fluid reference frame, yields the induction equation

$$
\partial \mathbf{B} / \partial t=\nabla \wedge(\mathbf{u} \wedge \mathbf{B})+\lambda \nabla^{2} \mathbf{B},
$$

where $\mathbf{u}(\mathbf{x}, t)$ stands for the velocity field and $\lambda$ for the magnetic diffusivity of the fluid. It is meaningful to note that the induction equation (1) bears a formal analogy with the vorticity equation, obtained by taking the curl of the NavierStokes equation in the case of a barotropic fluid with uniform density

$$
\partial \omega / \partial t=\nabla \wedge(\mathbf{u} \wedge \boldsymbol{\omega})+\nu \nabla^{2} \boldsymbol{\omega} .
$$

Here $\omega=\nabla \wedge \mathbf{u}$ denotes the vorticity field and $\nu$ the kinematic viscosity. However, because $\omega$ is related to $\mathbf{u}$, Eq. (2) is nonlinear while Eq. (1) is linear in $\mathbf{B}$.

Equations (1) and (2) are of the form of convectiondiffusion partial differential equations. The diffusion part is an invitation to connect the solution to the expectation value of some random walk. Indeed, let us remind that a FokkerPlanck equation, that is of the type of a diffusion equation, written (here in $1 \mathrm{D}$ for simplicity) as

$$
\frac{\partial P(x, t)}{\partial t}=-\frac{\partial}{\partial x}[F(x) P(x, t)]+\frac{1}{2} D \frac{\partial^{2} P(x, t)}{\partial x^{2}},
$$

describes the time evolution of the probability density function of the velocity of a particle under the influence of drag forces and random forces, as in Brownian motion, according to the Langevin equation

$$
\frac{d x}{d t}=F(x)+\eta(t)
$$

Here the noise $\eta$ is a Gaussian white noise with correlations $\left\langle\eta(t) \eta\left(t^{\prime}\right)\right\rangle=D \delta\left(t-t^{\prime}\right)$ and $P$ is the probability distribution

$$
P(x, t) \equiv\langle\delta(x-x(t))\rangle_{\eta},
$$

where $x(t)$ is the solution of the Langevin Eq. (4) and thus depends on the noise $\eta$, and $\langle\ldots\rangle_{\eta}$ is an average with respect to the noise.

Historically in this process, Chorin $^{8}$ produced a seminal work by proposing to solve the deterministic vorticity equation (2) in two space dimensions, that takes the form of a Fokker-Planck equation, through a stochastic approach. Recently, a stochastic formulation of the incompressible Navier-Stokes equation was derived in the thesis by Iyer $^{9}$ that rigorously provided an interpretation of viscous fluids as ideal inviscid fluids plus the Brownian motion. An historical account of the main results obtained between those two works may be found there. Lately, similar results have been derived in the incompressible amplified, magnetohydrodynamic (MHD), frame by Eyink. ${ }^{10}$ In particular, he showed that the viscous and resistive incompressible magnetohydrodynamic (MHD) equations were equivalent to having some stochastic conservation laws, and that similar results could be obtained in more refined non-ideal models, such as the Hall MHD and two-fluid plasma models with incompressible velocities.

The physical picture that emerges from those results in the frame of the induction equation (1) is the following. The magnetic field lines at any given initial time may be viewed as being frozen to the stochastic fluid flows and thus become themselves stochastic. The deterministic magnetic field at any point at a later time is the random ensemble average (or the expectation value) of the magnetic field vectors that are advected to that point by the stochastic flows. It is in this sense that it was written in the introduction that non-ideal effects, such as the resistive effects that are introduced in a phenomenological way through the Ohm's law, may be viewed as an implicit way to introduce stochasticity. This stochastic interpretation, related to the diffusion term in Eq. (1), takes place irrespective of the space dimension chosen in the modeling. However, the recurrent problem with this picture is that it predicts timescales for magnetic reconnection that are usually far longer than the realistic timescales, at least if one restricts the non-ideal effects simply to resistive effects, which is a crude assumption.

Indeed, let us consider the well-known (two-dimensional) Sweet-Parker model ${ }^{11,12}$ for magnetic reconnection using the frame of resistive magnetohydrodynamics. The Lundquist number, $S$, that denotes the order of magnitude of the ratio between the resistive to the Alfven timescale, is of the order $10^{14}$ in the solar corona. In this case, the timescale for magnetic reconnection obtained for the Sweet-Parker model is the geometric mean of the resistive and Alfven timescales, of the order of $10^{7} \mathrm{~s}$, that is of the order of three 
months, whereas the typical timescale for solar flares is about less than $1 \mathrm{~h}$.

Yet, it will now be shown that there exists a generic intrinsic stochasticity of magnetic field lines in 3D space that does not require the invocation of non-ideal effects.

\section{HAMILTONIAN FORMULATION OF MAGNETIC FIELD LINES}

Everyone who attended physics classes in high school has in mind a picture of magnetic field lines from the classical experiment of iron filings aligning on them after being sprinkled on a sheet of paper held over some magnet. It happens that, at each given time, these magnetic field lines may be viewed as trajectories of an Hamiltonian system. ${ }^{13,15}$ This comes from the single universal property of the magnetic field, namely, its divergence-free, or solenoidal, nature. This property is obviously more general than the induction equation, that is a MHD equation and thus relies on a fluid modeling of the plasma, although diluted hot plasma physics is generically kinetic, and on the Ohm's law that is a phenomenological approximation.

Considering an arbitrary parametrization $\mathbf{x}=\mathbf{x}(\lambda)$ $=\left(x^{1}(\lambda), x^{2}(\lambda), x^{3}(\lambda)\right)$ of the magnetic field lines, Cary and Littlejohn ${ }^{14}$ showed that the variational principle

$$
\delta \int d \lambda \mathbf{A}(\mathbf{x}) \cdot \frac{d \mathbf{x}}{d \lambda}=0,
$$

is identical to a dynamical variational principle. Indeed, the Cary-Littlejohn Lagrangian amounts to the Lagrangian of a massless particle in a magnetic field. Consequently, the equations of magnetic field lines, that are the resulting EulerLagrange equations

$$
(\nabla \times \mathbf{A}) \times \frac{d \mathbf{x}}{d \lambda}=\mathbf{B} \times \frac{d \mathbf{x}}{d \lambda}=\mathbf{0},
$$

were established to derive from a Hamiltonian system.

One can then choose the parameter $\lambda$ as a combination of the coordinates to eliminate it. For instance, if the field lines move in the direction of say, $x^{3}$, one may take $\lambda=x^{3}$. The magnetic field lines at each given time may then be viewed as trajectories of a Hamiltonian system $H$ of the (non-canonical) variables $\left(x^{1}, x^{2}, x^{3}\right)$.

Yet, the chaos theory tells us that the minimal number of degrees of freedom required then for the possible emergence of chaos is three. Therefore, two-dimensional models, or models in which the effective dimension is reduced due to some symmetry, can only have integrable magnetic field lines, whereas three-dimensional models may potentially exhibit some spatially chaotic magnetic field lines.

The fact that magnetic field lines are generically chaotic in $3 \mathrm{D}$ space has important consequences in the representation of the problem of magnetic reconnection: a magnetic field line in a bounded 3D domain may wander and never close. Consequently, the classical smooth pictures of $2 \mathrm{D}$ projections of the reconnection of magnetic field lines could be wrong and misleading. Generic 2D Poincaré sections of magnetic field lines should generically display some stochastic component.
Let us finally comment on the role of the geometry. In toroidal devices for magnetic confinement fusion, magnetic field lines are dominantly along the toroidal direction and the poloidal cross-sections form natural Poincaré sections. This is obviously no longer valid usually in the astrophysical context. However, the absence of natural cross-sections does not affect the validity of the Hamiltonian formulation of magnetic field lines coming from the divergence-free property of the magnetic field. As emphasized in Ref. 16, the Hamiltonian description of magnetic field lines is general and can be obtained independent of the geometry of field lines or the particular coordinate system used. The Hamiltonian formalism is useful in that it allows to connect to some of the nicest achievements of twentieth century's mathematical physics, accompanying the KolmogorovArnold-Moser theory, some of which emanating from the plasma physicists (the Reader is referred to Ref. 17 for a recent review).

\section{THE EXAMPLE OF SAWTEETH}

We shall now turn to the example of sawteeth in tokamak plasmas to investigate the impact of the spatial chaos of magnetic field lines on the reconnection features. Some evidence of the existence of spatially chaotic magnetic field lines in the collapse reconnection phase in sawteeth has been presented elsewhere through extensive numerical studies. ${ }^{21}$ Indeed, using a realistic frame for the time evolution of the electromagnetic field in sawteeth, our numerical results pointed to the necessity of having stochastic magnetic field lines to reproduce the experimental evolution of some heavy test particles during the reconnection phase. Our focus will be here on the impact of this spatial chaos on the dynamical behaviour and acceleration of charged particles during the sawtooth crash.

\section{A. Electromagnetic description of the magnetic reconnection in tokamaks}

In the toroidally shaped fusion devices, the double poloidal and azimuthal periodicity is used to Fourier decompose the fields on the $m$ poloidal and $n$ toroidal components. The winding of the equilibrium axisymmetric magnetic field as a function of the minor radius, $r$, is measured by the socalled $q$-profile, with $q(r) \simeq r / R \times B_{t o r} / B_{p o l}$, with $R$ denoting the major radius. Sawtooth cycles are initiated in situations where the tokamak plasmas become linearly unstable to the $m=1, n=1$ internal mode. This necessitates that the $q$-profile be less than one in the plasma center. In the typical course of a sawtooth cycle, the $n=1$ mode appears first, being linearly triggered, with the dominant poloidal harmonics $m=1$. This means that the associated magnetic island starts to grow. Then, $n=2$ and later $n=3$ modes are nonlinearly triggered. ${ }^{18,19}$

Much of the ignorance and incompleteness of the understanding of magnetic reconnection comes from the difficulty to diagnose it with a sufficient resolution either in space or in laboratory magnetic confinement devices. For instance, in tokamak plasmas, it is only possible to access the experimental to the toroidal $(n)$ spectrum of the magnetic perturbations without resolving it poloidally. Numerical simulations of 
reconnection in astrophysical plasmas and sawteeth are also notably difficult since these problems involve a wide range of space and time scales. Because our objective is to single out the effect of the spatial chaos of the magnetic field lines, we have used a different framework. From the electromagnetic point of view, the sawtooth collapse phase is associated to an abrupt variation of the magnetic field perturbation for some tens of Alfvèn times. This collapse phase will be modeled using two electromagnetic sets: one in which helical symmetry is assumed and only $m=n$ modes are retained making the problem two-dimensional and the one in which one allows for some additional $m \neq n$ modes so that the problem is truly three-dimensional and the stochasticity may show up above some thresholds in the mode amplitudes. The associated perturbations of the electric field have been computed using an ideal MHD hypothesis. This ideal treatment for the electric field is an approximation to the real electric field that enables to have the magnetic perturbations as the single set of control parameters, so that we can focus on the impact of the magnetic chaos. Indeed the flow velocity $\mathbf{u}(\mathbf{r}, t)$ that serves to express the electric field as $\mathbf{E}(\mathbf{r}, \mathbf{t})=-\mathbf{u}(\mathbf{r}, t) \times \mathbf{B}(\mathbf{r}, t)$ may be related to the time derivative of the mode displacements $\xi^{m, n}(\mathbf{r}, t)$ that parameterize the magnetic field lines.

In this framework, extensive numerical simulations of the time evolution of collections of test particles during the sawtooth collapse phase have been performed ${ }^{20,21}$ solving the equations of motion

$$
m \frac{d^{2} \mathbf{r}}{d t^{2}}=q\left(\frac{d \mathbf{r}}{d t}-\mathbf{u}(\mathbf{r}, t)\right) \times \mathbf{B}(\mathbf{r}, t) .
$$

In the present study, the test particles, of mass $m$ and charge $q$, were either impurity ions or protons. We chose to use here, the nickel ions as impurity ions to reproduce the conditions of some tokamak experiments. ${ }^{22}$

\section{B. The two classes of magnetic models}

As previously said, two classes of models for the time behavior of the magnetic mode amplitudes $\xi_{0}^{m n}(t)$ around the sawtooth crash were chosen.

In the first class (C2D), that serves as a reference, only modes having the same helicity have been retained. The conservation of helicity ensures that the effective dimensionality of these models is two: magnetic field lines derive from Hamiltonians depending solely on a radial variable and on the helical angle $\theta-\phi$.

In the second class of models (C3D), this restriction is lifted, and the effective dimensionality is three. Practically speaking, the $(m, n)$ modes involved are the $(1,1)$ and $(2,2)$ modes in C2D models while they are the $(1,1),(2,2),(2,1)$, $(3,2)$, and $(4,3)$ modes in C3D models, the last two modes being subdominant compared to $(2,1)$. The two classes of models are represented in Figure 2. Poincaré sections are used to probe the chaos of the magnetic field lines at some given time.

\section{From Eulerian magnetic to Lagrangian particle chaos}

The spatial chaos of the magnetic field lines may not be sufficient to imply that the motion of charged particles in these fields is chaotic. Therefore it is desirable to quantify chaos at the level of the charged particles and answer the question: How do the chaotic properties of the magnetic field lines transfer to the plasma particles during the sawtooth crash? As shown below, a proper indicator will be provided by the local (maximal) finite-time Lyapunov exponent (FTLE). Let us note that this is only a qualitative indicator of chaos in the sense that the true indicator, namely, the maximum Lyapunov exponent being strictly positive, is only defined asymptotically in time. A FTLE of an integrable system may appear strictly positive due to its evaluation in a finite time. In the numerical simulations, we used the nickel ions as charged test-particles to compute the FTLE in both an integrable (C2D) and a chaotic (C3D) model of magnetic field lines for the sawtooth reconnection collapse phase.

At time $t_{0}=0$, let us consider an arbitrary point $\mathbf{r}_{0}$. A nickel particle put initially in this point will be located after the crash time $T$ at a position $\mathbf{r}\left(\mathbf{r}_{0}, \mathbf{v}_{0}, T\right) \equiv \Lambda_{\mathbf{v}_{0}}^{T}\left(\mathbf{r}_{0}\right)$ obtained from the integration of the equation of motion (6) and the additional data of the initial velocity $\mathbf{v}_{0}$. For clarity, let us assume that at time $t_{0}$ all particles have the same velocity $\mathbf{v}_{0}$, so that one can use the short-hand notation $\Lambda^{T}\left(\mathbf{r}_{0}\right)$. Let us consider any arbitrarily oriented infinitesimal displacement $\delta$ $\mathbf{r}_{0}$. A particle put initially at the point $\mathbf{r}_{0}+\delta \mathbf{r}_{0}$ will have deviated after a time interval $T$ from a particle initially put at point $\mathbf{r}_{0}$ by the perturbation vector (up to $O\left(\left\|\delta \mathbf{r}_{0}\right\|^{2}\right.$ ) second order terms)

$$
\delta \mathbf{r}(T)=\Lambda^{T}\left(\mathbf{r}_{0}+\delta \mathbf{r}_{0}\right)-\Lambda^{T}\left(\mathbf{r}_{0}\right)=\frac{d \Lambda^{T}\left(\mathbf{r}_{0}\right)}{d \mathbf{r}} \delta \mathbf{r}_{0} .
$$

Its norm is then $\|\delta \mathbf{r}(T)\|=\left\langle\delta \mathbf{r}_{0}, M\left(\mathbf{r}_{0}, T\right) \delta \mathbf{r}_{0}\right\rangle^{1 / 2}$, where

$$
M\left(\mathbf{r}_{0}, T\right) \equiv\left(\frac{d \Lambda^{T}\left(\mathbf{r}_{0}\right)}{d \mathbf{r}}\right)^{\dagger} \frac{d \Lambda^{T}\left(\mathbf{r}_{0}\right)}{d \mathbf{r}}
$$

is a symmetric matrix. If $\lambda_{\max }^{M\left(\mathbf{r}_{0}, T\right)}$ denotes its larger eigenvalue, then the FTLE at point $\mathbf{r}_{0}$ with a finite integration time $T, \sigma^{T}\left(\mathbf{r}_{0}\right)$, is defined through

$$
\max _{\delta \mathbf{r}_{0} \neq 0} \frac{\|\delta \mathbf{r}(T)\|}{\left\|\delta \mathbf{r}_{0}\right\|}=\sqrt{\lambda_{\text {max }}^{M\left(\mathbf{r}_{0}, T\right)}} \equiv e^{\sigma^{T}\left(\mathbf{r}_{0}\right) T} .
$$

The numerical computation of the FTLE field is shown in Fig. 3. For the parameters chosen, it follows from the inspection of Figure 3 that two nickel particles initially separated by say, $1 \mathrm{~mm}$ will be separated after the $200 \mu$ s (near-)crash phase by at most a few centimeters with the non-chaotic model (a) of $\mathbf{B}$ lines, versus by distances as large as $20 \mathrm{~m}$, that is a toroidal excursion, in the chaotic case (b). In the case of integrable magnetic field lines, the FTLE is then globally smaller than in the case of chaotic magnetic field lines. The local maxima of the FTLE field in the 2D case with integrable magnetic field lines come from the region of the magnetic x-point and separatrices.

Let us explore more closely the connection between the Eulerian properties of the magnetic field and the Lagrangian properties of the particle motions. The linearization of the equations of motion (6) about some reference trajectory $\mathbf{r}(t)$ yields 

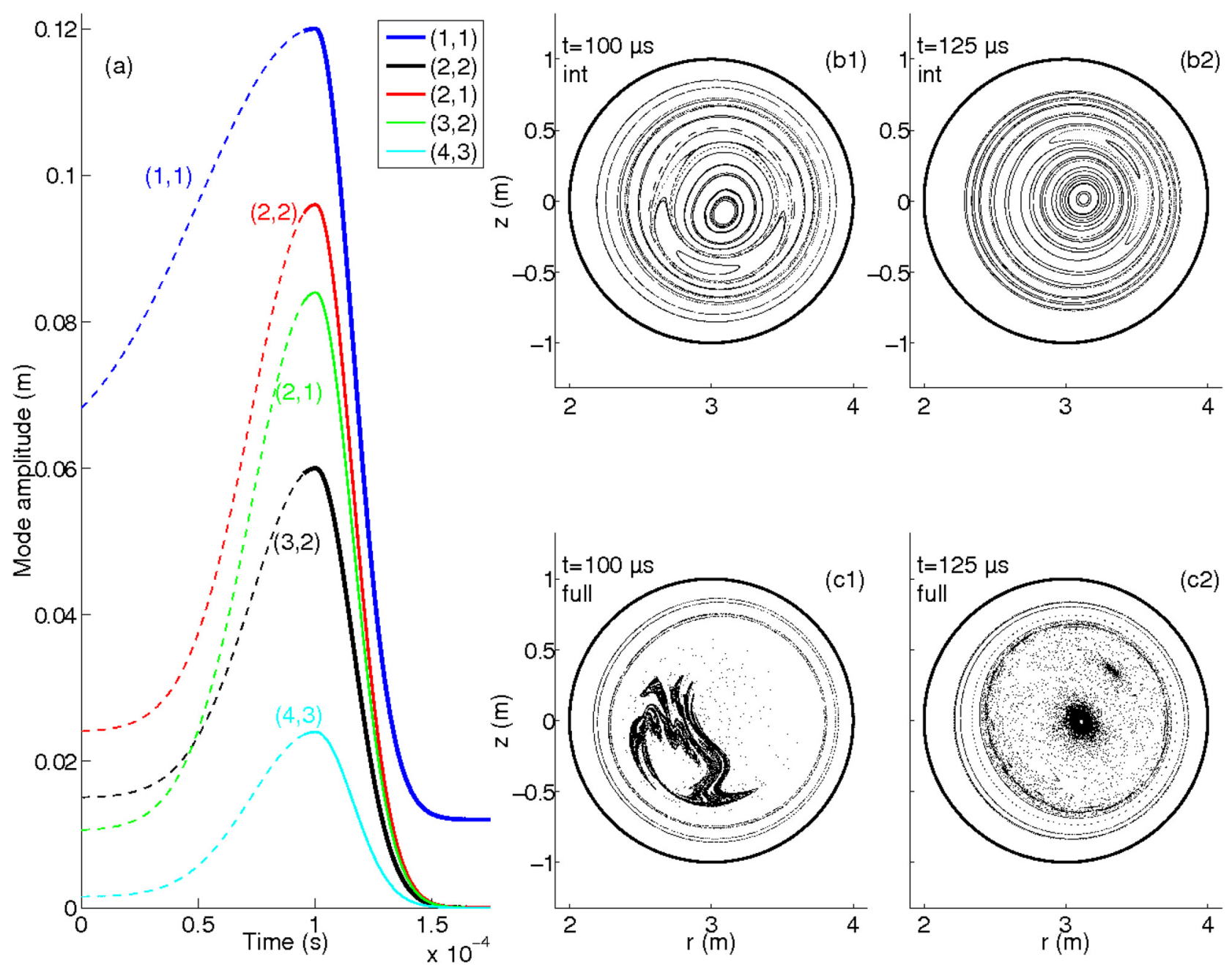

FIG. 2. (a) Model for the time behavior of the mode amplitudes $\xi_{0}^{m n}(t)$ around the sawtooth crash. The two-dimensional integrable model (C2D) consists in retaining only the $(1,1)$ and $(2,2)$ modes. The three-dimensional model (C3D) takes also the $(2,1),(3,2)$, and $(4,3)$ modes. Poincaré sections of the $\mathbf{B}$ lines in the integrable scenario are plotted in (b1) at the crash onset when $t=100 \mu \mathrm{s}$, (b2) in the middle of the crash for $t=125 \mu \mathrm{s}$. The corresponding plots for the stochastic C3D model are in (c1) and (c2).

$$
\begin{aligned}
m \frac{d^{2} \delta \mathbf{r}}{d t^{2}}= & q \frac{d \delta \mathbf{r}}{d t} \times \mathbf{B}(\mathbf{r}, t)+q\left(\frac{d \mathbf{r}}{d t}-\mathbf{u}(\mathbf{r}, t)\right) \times\left.\delta \mathbf{r} \cdot \nabla\right|_{(\mathbf{r}, t)} \mathbf{B} \\
& -\left.q \delta \mathbf{r} \cdot \nabla\right|_{(\mathbf{r}, t)} \mathbf{u} \times \mathbf{B}(\mathbf{r}, t) .
\end{aligned}
$$

In the right hand side of Eq. (10), the second term involves the Jacobian of the magnetic field and the third term, the Jacobian of the velocity field. In 3D, the magnetic field lines, as well as the velocity field lines, may become spatially chaotic. This Eulerian chaos of velocity field lines is sometimes taken in the fluid mechanics community as the definition of turbulence. ${ }^{23,24}$ In this case, space gradients are of the order of the local spatial exponentiation rates of these fields. In 2D, these are null, yet some space discontinuities
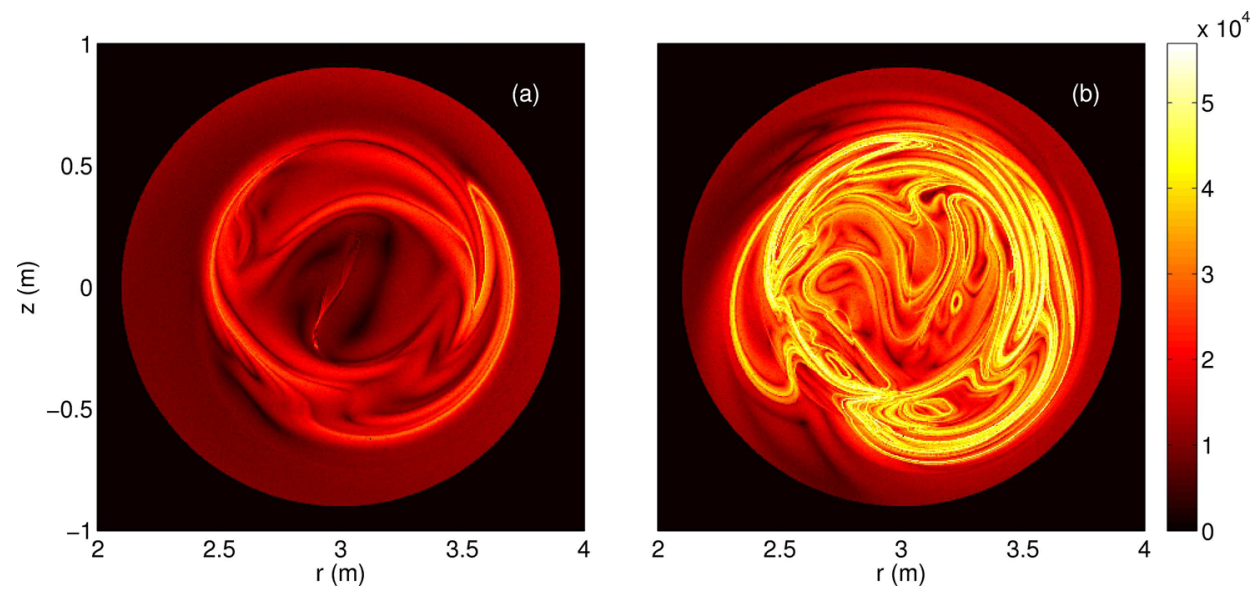

FIG. 3. Numerical FTLE $\sigma^{T}$ field for the nickel motion plotted in a poloidal cross-section using (a) the integrable and (b) the stochastic models for magnetic field lines, as shown in Fig. 2, are associated to the crash phase. Time zero is chosen as in Fig. 2(a) and $T=200 \mu$ s. The scale unit is $\mathrm{s}^{-1}$. 
(e.g., due to shocks) associated with large space gradients may promote the spreading of the charged particles whatever the space dimension.

\section{IMPACT OF THE CHAOS OF MAGNETIC FIELD LINES ON RECONNECTION OBSERVABLES}

\section{A. Chaos or stochasticity?}

Let us start by some clarifying point. It is sufficient for the magnetic field lines to be locally chaotic that a small subset of magnetic modes be destabilized. As the amplitudes of these modes grow, the Chirikov resonance-overlap ${ }^{25}$ eventually occurs, signifying the onset of large scale chaos between the nonlinear resonances. ${ }^{26}$ One usually speaks of stochasticity when there is a large spectrum of excited modes and when the phase space does no longer possess any remnant of coherent structures. Stochasticity refers to pure noise whereas the phase space of chaotic systems may be modeled by a mixture of stochastic and coherent components. It is however sufficient to have the chaotic magnetic and/or velocity field lines to impact the evaluation of macroscopic observables. In order to motivate this statement, let us, for instance, consider the rate of energy transfer between the magnetic field and the plasma.

\section{B. Justification for an increased reconnection rate with chaotic B lines vs. the regular B lines}

The rate of energy transfer from the magnetic field to the plasma at time $t$ is

$$
\begin{aligned}
s(t) & =\iiint \mathbf{j}(\mathbf{r}, t) \cdot \mathbf{E}(\mathbf{r}, t) d^{3} x \\
& =\frac{1}{\mu_{0}} \iiint \mathbf{u}(\mathbf{r}, t) \cdot[\mathbf{j}(\mathbf{r}, t) \times \mathbf{B}(\mathbf{r}, t)] d^{3} x,
\end{aligned}
$$

using the ideal MHD approximation. Approximating the plasma equation of motion by the collisionless MHD equation

$$
\rho \frac{d \mathbf{u}(\mathbf{r}, t)}{d t}=\mathbf{j}(\mathbf{r}, t) \times \mathbf{B}(\mathbf{r}, t)-\nabla p(\mathbf{r}, t),
$$

we have then

$$
\begin{aligned}
s(t) & =\frac{1}{\mu_{0}} \iiint \mathbf{u}(\mathbf{r}, t) \cdot\left(\rho \frac{d \mathbf{u}(\mathbf{r}, t)}{d t}+\nabla p(\mathbf{r}, t)\right) d^{3} x \\
& =\frac{d}{d t}\left(\frac{1}{\mu_{0}} \iiint \frac{1}{2} \rho u^{2} d^{3} x\right)+\frac{1}{\mu_{0}} \iiint \mathbf{u} \cdot \nabla p d^{3} x .
\end{aligned}
$$

Our aim is to compare the rate of energy transfer from the magnetic field to the plasma in the case where the magnetic field lines are chaotic, so that the Hamiltonian model governing the magnetic field lines possesses some ergodic component (or chaotic sea), with the case where the magnetic field lines are regular.

In doing this comparison, we consider a stage in the reconnection process at the border of chaos, i.e., just prior to the onset of nonlinearities. Then the convective term $(\mathbf{u} \cdot \nabla) \mathbf{u}$ remains negligible, and the inertia term may be neglected. ${ }^{18}$ We consider this stage when the displacement $\xi$ keeps growing at a constant rate (with $\mathbf{u} \equiv \partial \xi / \partial t$ constant) and the Laplace force is balanced by the pressure gradient in (11). Then we consider the two options for the subsequent evolution of the system. One in which we allow for the (local) emergence of the chaos of magnetic field lines in the reconnection zone and the one in which we assume that magnetic field lines remain regular everywhere.

Let us denote by $s_{c}(t)$ the rate of energy transfer in the first chaotic configuration and by $s_{r}(t)$ its counterpart in the regular case. Basically, we shall assume that magnetic field lines are almost identical in both cases except within the reconnection zone, denoted by $\mathcal{V}_{\text {rec }}$, in which the magnetic chaos may develop about the separatrices, and we shall neglect the possible discrepancies in the velocity field $\mathbf{u}(\mathbf{r}, t)$ between the two cases. We have then

$$
s_{c}(t)-s_{r}(t)=\iiint_{\mathcal{V}_{\text {rec }}} \mathbf{u}(\mathbf{r}, t) \cdot\left\{\left.\nabla p(\mathbf{r}, t)\right|_{c}-\left.\nabla p(\mathbf{r}, t)\right|_{r}\right\} d^{3} x .
$$

At equilibrium, Eq. (11) yields

$$
\mathbf{B}(\mathbf{r}, t) \cdot \nabla p(\mathbf{r}, t)=0 .
$$

The fulfilment of this equality requires that the pressure gradient vanishes identically in the space region of the magnetic ergodic component, meaning that the pressure be constant in this region. (Let us note that this is indeed in agreement with the results of some sophisticated experimental tokamak diagnostics that do indicate that the pressure (and electron temperature) profiles flatten in the reconnection zone just before the sawtooth collapses. ${ }^{27}$ ) In this situation, the difference (12) between the rates of energy transfer to the plasma in the chaotic versus the regular magnetic cases becomes

$$
s_{c}(t)-s_{r}(t) \simeq-\left.\iiint_{\mathcal{V}_{\text {rec }}} \mathbf{u}(\mathbf{r}, t) \cdot \nabla p(\mathbf{r}, t)\right|_{r} d^{3} x .
$$

In the reconnection region $\mathcal{V}_{\text {rec }}$ (see Fig. 1), the transverse gradients are large and the plasma gradients must remain finite to be physical. The velocity $\mathbf{u}$ is dominantly and rigidly directed towards the reconnection layer zone, that is in the direction opposite to the magnetic island. The plasma pressure is high in the tokamak core and low about the tokamak edges, so that the gradient pressure is in the direction opposite to the velocity $\mathbf{u}$ in the domain $\mathcal{V}_{\text {rec }}$. This leads to the prediction, derived within the sawtooth example, that the introduction of some local chaos of the magnetic field lines, inducing the creation of some ergodic component, enhance the rate of energy transfer from the magnetic field to the plasma with

$$
s_{c}(t)>s_{r}(t)
$$

\section{Particle acceleration in the collapse reconnection phase}

Some numerical simulations on large collections of test charged particles have been performed in order to measure the variation of particle energies in various electromagnetic 
(a)

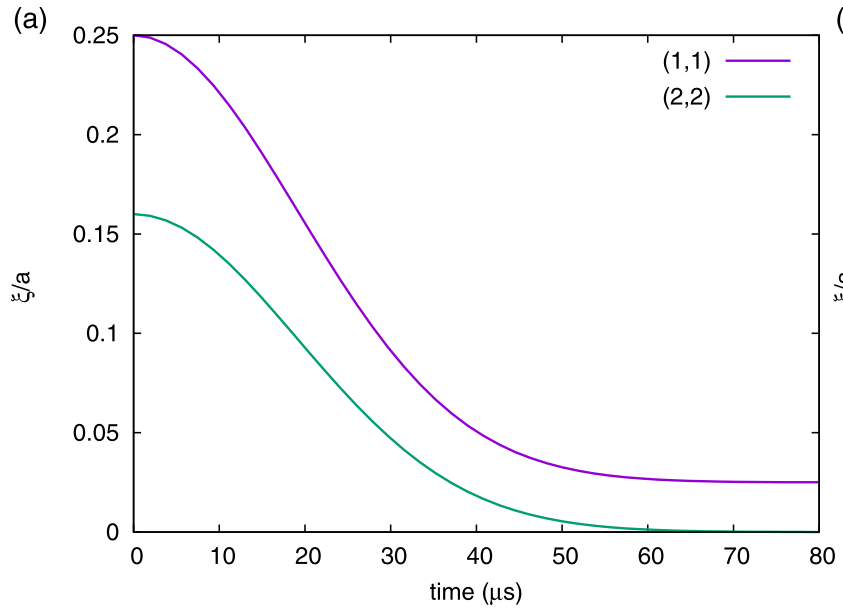

(b)

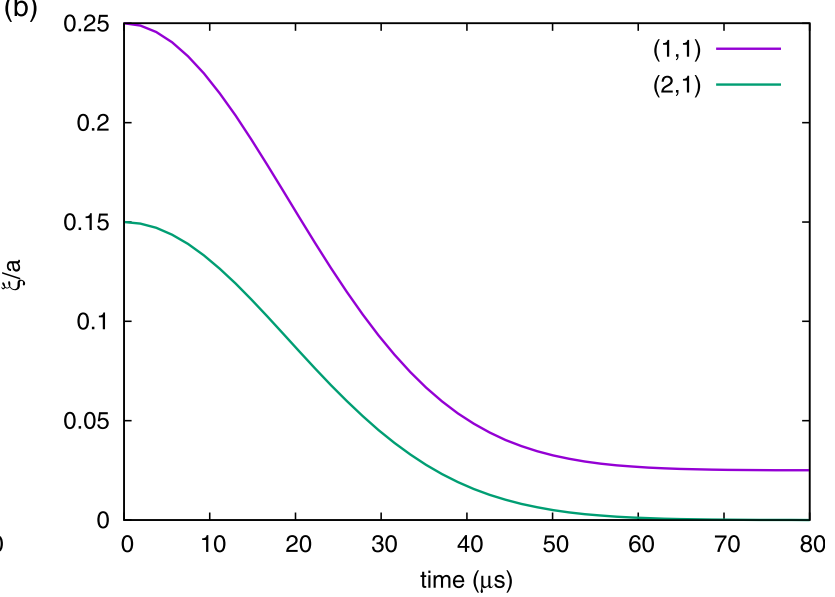

FIG. 4. Time evolution of the magnetic mode amplitudes during the sawtooth reconnection collapse with a magnetic perturbation composed of (a) (1, 1) and $(2,2)$ modes, (b) $(1,1)$ and $(2,1)$ modes. In both cases, the magnitude of the magnetic field perturbation $\delta B / B$ is identical.

models during the sawtooth collapse phase. As already said, the timescale for the sawtooth collapse in tokamaks is of the order of $100 \mathrm{~ms}$.

In order to measure the impact of the chaos of magnetic field lines, all other things being equal, we used as, in Sec. IV, two different sets of magnetic field lines with the same amount of non-axisymmetric magnetic perturbation $\delta B / B$ in both cases. More precisely, in a first class of models (C2D), the integrability of magnetic field lines is ensured by using an electromagnetic field perturbation with only $m=n$ modes, typically consisting of the $(1,1)$ mode and possibly also of the $(2,2)$ mode. In the second class of models (C3D), the electromagnetic model for the non-axisymmetric perturbation includes $(m, n)$ modes with $m \neq n$, so that the magnetic field lines become globally chaotic above some thresh$\operatorname{old}^{25,26}$ in the magnetic field perturbation $\delta B / B$. In the case when the $m=n=1$ and $m=2, n=1$ modes are retained, this occurs when the magnetic islands associated to the $(1,1)$ mode and to the $(2,1)$ mode overlap. Before this threshold in the (C3D) cases, there exists already some phase space zones, bounded by KAM tori, with ergodic components in the region of the separatrices and mostly about the x-points. This means that, within a three-dimensional modeling, the chaos of magnetic field lines linked to the excitation of plasmoids first emanates in the reconnection zone. Typically the area of the chaotic sea or ergodic component is a growing function of $\delta B / B$ in this case.

Figure 4 represents the time evolution of the modes composing the magnetic perturbation associated to the tokamak sawtooth crash with a) integrable magnetic field lines and b) with some chaos of magnetic field lines in the reconnection region ( $q=1$ surface). In those two electromagnetic models for the sawtooth collapse, the dynamics of $10 \times 10^{6}$ test protons have been integrated being initially and uniformly distributed in the reconnection zone (about $q=1$ ). At initial time, i.e., just before the sawtooth collapse, all the protons were taken to have the same energy $E_{0}=4 \mathrm{keV}$, and their initial velocities were isotropically distributed.

The energy distribution of protons at the end of the sawtooth collapse is represented in Figure 5. Despite the smallness of the duration of the collapse phase, this figure shows a significant difference in the energy distribution of protons at the end of the collapse between the magnetic integrable and the chaotic cases. In the chaotic (b) case, the variance of the energy distribution is measured to be about three times larger than in the integrable (a) case. This means that protons have been more heated during the collapse phase in the case with chaotic magnetic field lines.

If the magnetic mode amplitudes are reduced, being for instance reduced to the fourth of the case just considered in Figure 4, this differential heating is strongly attenuated as seen in Figure 6. Figure 7 shows the intermediary case in which the mode amplitudes of Figure 4 are rescaled by one half. On all cases, the variance of the energy distribution is always larger in the case (b) with a magnetic perturbation composed of the $(1,1)$ and $(2,1)$ modes compared with the integrable case a) with the $(1,1)$ and $(2,2)$ modes, although the ratio of the perturbation $\delta B / B$ is the same in both cases. In the case of Figure 6, there is no large scale chaos, i.e., no resonance overlap between the $(1,1)$ and $(2,1)$ modes and the ratio of the energy variance between case (b) and case

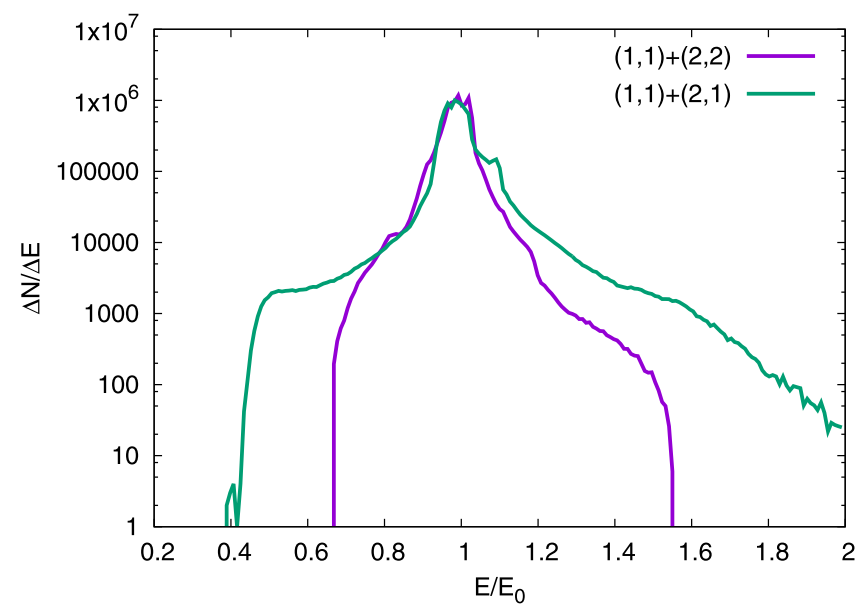

FIG. 5. At the onset of the collapse ( $t=0$ in Fig. 4), an ensemble of protons are uniformly distributed in the reconnection region (about the $q=1$ surface) all having the same energy $E_{0}$. Displayed in the Figure is their energy distribution at the end of the sawtooth collapse for the cases (a) and (b) of Fig. 4. The scale is logarithmic along the $y$-axis. $10 \times 10^{6}$ of protons have been used. 


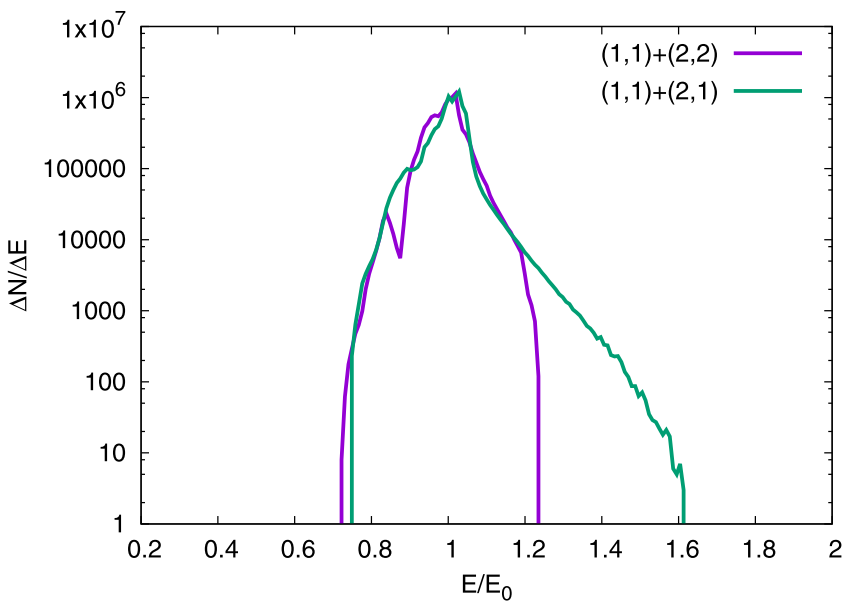

FIG. 6. Same as in Figure 5 for one fourth of the magnetic mode amplitudes of Figure 4 with a collection of ten millions of protons.

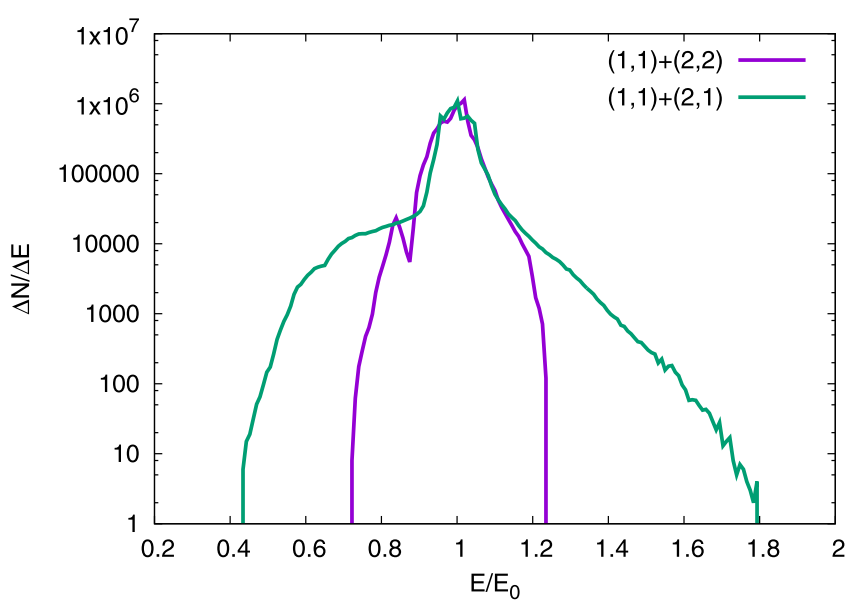

FIG. 7. Same as in Figure 5 for one half of the magnetic mode amplitudes of Figure 4 with a collection of $10 \times 10^{6}$ of protons.

(a) is 1.5 . This ratio goes to 2.2 in Figure 7 and to 2.8 in Figure 5 suggesting a noticeable impact of the chaos of magnetic field lines on particle heating during the collapse reconnection phase.

\section{CONCLUSION}

The present study has been devoted to the relationships between magnetic reconnection and stochastic properties. In the first part, it has been recalled that the dissipative term associated with plasma resistivity in the evolution (induction) equation of the magnetic field could be equivalently interpreted as a stochastic (noise) term in a corresponding Langevin equation approach for the evolution of magnetic field lines. However, in most physical situations, the timescales given by a simple resistive interpretation of magnetic reconnection are far larger than the measured ones so that there is a general agreement that one needs to search for additional ingredients to explain the fastness of magnetic reconnection.

Leaving apart the potential non-ideal effects other than resistivity, we have introduced in the second part of this study another source of stochasticity, coming from the spatial (or Eulerian) chaos of magnetic field lines. This may only show up in a three-dimensional description of magnetic reconnection, since any two-dimensional description will force the integrability of magnetic field lines. In the situation of spontaneous magnetic reconnection, in which reconnection initiates from an instability and the formation of plasmoid(s), the reconnection region contains magnetic x-points and is prone to the development of chaos. The impact of this introduction of chaotic magnetic field lines in the reconnection zone has been investigated. A general theoretical estimate of differences of the rate of energy transfer from the magnetic field to the plasma between the cases of integrable and chaotic magnetic field lines has been presented. This indicates that the rate of energy transfer is larger when magnetic field lines become chaotic in the reconnection zone compared to the integrable case.

Simulations also reveal that the chaotic magnetic field lines can efficiently mix charged particles so that there must be an intimate connection in plasmas undergoing magnetic reconnection between turbulence at the fluid level (i.e., velocity field being non laminar) and turbulence in the magnetic field (magnetic field lines being chaotic at each given time).

Finally, the tokamak sawteeth forms a unique frame in which a conclusive answer to the longstanding problem of the fastness of (spontaneous) magnetic reconnection could someday be reached. Even if the reconnection zone is tiny which makes in-situ measurements almost impossible, contrarily to space situations, the space compactness of the process naturally solves the difficult problem of the boundary conditions in the astrophysical situations. A dialog between the magnetic reconnection communities in astrophysical and laboratory plasmas should certainly push forward the field. The implication of the results presented here to the recent outcomes of some 3D magnetic reconnection dedicated experiment ${ }^{28}$ should also be explored.

\section{ACKNOWLEDGMENTS}

Some financial support from the ECOS-MINCyT Research Grant No. A09E02 is gratefully acknowledged. This work was carried out within the framework of the French Research Federation for Fusion Studies.

${ }^{1}$ R. G. Giovanelli, Nature 158, 81 (1946).

${ }^{2}$ J. Birn and E. R. Priest, Reconnection of Magnetic Fields (Cambridge University Press, Cambridge, UK, 2007).

${ }^{3}$ E. G. Zweibel and M. Yamada, Annu. Rev. Astron. Astrophys. 47, 291-332 (2009).

${ }^{4}$ V. Angelopoulos, J. P. McFadden, D. Larson, C. W. Carlson, S. B. Mende, H. Frey, T. Phan, D. G. Sibeck, K.-H. Glassmeier, U. Auster et al., Science 321, 931-935 (2008).

${ }^{5}$ S. Von Goeler, W. Stodiek, and N. Sauthoff, Phys. Rev. Lett. 33, 1201 (1974).

${ }^{6}$ R. von Steiger, Front. Phys. 1, 6 (2013).

${ }^{7}$ A. Retinò, D. Sundkvist, A. Vaivads, F. Mozer, M. André, and C. J. Owen, Nat. Phys. 3, 235-238 (2007).

${ }^{8}$ A. J. Chorin, J. Fluid. Mech. 57, 785-796 (1973).

${ }^{9} \mathrm{G}$. Iyer, "A stochastic Lagrangian formulation of the incompressible Navier-Stokes and related transport equations, Ph.D. thesis," (University of Chicago, 2006).

${ }^{10}$ G. L. Eyink, J. Math. Phys. 50, 083102 (2009).

${ }^{11}$ E. N. Parker, J. Geophys. Res. 62, 509, doi:10.1029/JZ062i004p00509 (1957).

${ }^{12}$ P. A. Sweet, in Electromagnetic Phenomena in Cosmical Physics, edited by B. Lehnert (Cambridge University Press, New York, 1958), p. 123. 
${ }^{13}$ A. H. Boozer, Phys. Fluids 26, 1288 (1983).

${ }^{14}$ J. R. Cary and R. G. Littlejohn, Ann. Phys. 151, 1-34 (1983).

${ }^{15}$ P. J. Morrison, Phys. Plasmas 7, 2279-2289 (2000).

${ }^{16}$ M. S. Janaki and G. Ghosh, J. Phys. A: Math. Gen. 20, 3679-3685 (1987).

${ }^{17}$ D. F. Escande, Plasma Phys. Controlled Fusion 58, 113001 (2016).

${ }^{18}$ M.-C. Firpo and B. Coppi, Phys. Rev. Lett. 90, 095003 (2003).

${ }^{19}$ M.-C. Firpo, W. Ettoumi, R. Farengo, H. E. Ferrari, P. L. García-Martínez, and A. F. Lifschitz, Phys. Plasmas 20, 072305 (2013).

${ }^{20} \mathrm{~W}$. Ettoumi, see http://www.theses.fr/2013EPXX0100 for thèse de l'Ecole Polytechnique (2013)

${ }^{21}$ M.-C. Firpo, A. F. Lifschitz, W. Ettoumi, R. Farengo, H. E. Ferrari, and P. L. García-Martínez, "Evidence and relevance of spatially chaotic magnetic field lines in MCF devices" (unpublished).

${ }^{22}$ J. A. Wesson, B. Alper, A. W. Edwards, and R. D. Gill, Phys. Rev. Lett. 79, 5018 (1997).
${ }^{23}$ E. J. Hinch, "Mixing: Turbulence and chaos - An introduction," in Mixing: Chaos and Turbulence, NATO ASI Series Vol. 373, edited by H. Chaté, E. Villermaux, and J.-M. Chomaz, pp. 37-56 (Kluwer Academic/Plenum, 1999).

${ }^{24} \mathrm{As}$ the Eulerian chaos of velocity field lines can only exist in 3D, two-dimensional turbulence may then be called a misuse/abuse of language.

${ }^{25}$ B. V. Chirikov, J. Nucl. Energy Part C: Plasma Phys. 1, 253 (1960) [At. Energ. 6, 630 (1959) (in Russian)].

${ }^{26}$ D. F. Escande, Phys. Rep. 121, 165 (1985)

${ }^{27}$ V. S. Udintsev, M. Ottaviani, P. Maget, G. Giruzzi, J.-L. Ségui, T. Aniel, J. F. Artaud, F. Clairet, M. Goniche, G. T. Hoang et al., Plasma Phys. Controlled Fusion 47, 1111 (2005).

${ }^{28}$ W. Gekelman, T. De Haas, W. Daughton, B. Van Compernolle, T. Intrator, and S. Vincena, Phys. Rev. Lett. 116, 235101 (2016). 